\title{
Diasporas et Espaces transnationaux, Bruneau Michel
}

Paris, Economica (Anthropos Ville), 2004, 249 p., ISBN : 271784760X

\section{William Berthomière}

\section{(2) OpenEdition}

Journals

Édition électronique

URL : https://journals.openedition.org/remi/4274

DOI : $10.4000 /$ remi. 4274

ISSN : $1777-5418$

Éditeur

Université de Poitiers

Édition imprimée

Date de publication : 1 juin 2005

Pagination : 165-166

ISBN : 2-911627-40-7

ISSN : 0765-0752

Référence électronique

William Berthomière, "Diasporas et Espaces transnationaux, Bruneau Michel », Revue européenne des migrations internationales [En ligne], vol. 21 - n² | 2005, mis en ligne le 01 octobre 2008, consulté le 15 avril 2022. URL : http://journals.openedition.org/remi/4274 ; DOI : https://doi.org/10.4000/remi.4274

Ce document a été généré automatiquement le 15 avril 2022.

(c) Université de Poitiers 


\title{
Diasporas et Espaces transnationaux, Bruneau Michel
}

Paris, Economica (Anthropos Ville), 2004, 249 p., ISBN : 271784760X

\author{
William Berthomière
}

\section{RÉFÉRENCE}

Diasporas et Espaces transnationaux, Bruneau Michel, Paris, Economica (Anthropos Ville), 2004, 249 p., ISBN : $271784760 X$

1 L'ouvrage de Michel Bruneau s'inscrit dans un ensemble de publications en langue française qui vise à synthétiser et à conceptualiser les recherches empiriques qui ont été réalisées au fil des quinze dernières années. Au cours de cette période, le thème des diasporas a pris une place de choix au sein des sciences sociales notamment avec l'importante production anglo-saxonne issue de programmes de recherche comme Transnational Communities (financé par l'ESRC britannique). Ainsi dans la lignée des travaux précurseurs de Gabriel Sheffer, William Safran, Robin Cohen, Georges Prévélakis, Kachig Tölölyan ou plus récemment Steven Vertovec, Michel Bruneau a choisi de livrer un ouvrage transversal sur le thème en privilégiant un point de vue disciplinaire, celui de la géographie.

2 Pour mener à bien cette synthèse, l'auteur a choisi - comme a pu le faire Stéphane Dufoix dans un récent ouvrage sur le même thème ${ }^{1}-$ de concentrer sa réflexion sur la question de la définition de la notion même de diaspora, puis de décliner les concepts et thématiques qui composent la «nébuleuse » de recherches offerte par cette notion.

L'ouvrage s'organise en un ensemble de sept chapitres qui ont pour fil conducteur la dispersion, évènement fondateur des diasporas. De proche en proche, la dispersion mène Michel Bruneau de la question d'une nécessaire définition du phénomène observé à celles de l'appartenance et de la mémoire puis à celle des réseaux en intégrant la dispersion comme ressource « spatiale » (comme capital social). Dans ses deux derniers chapitres, l'auteur termine la boucle de sa réflexion en effectuant d'une part, un retour 
sur le premier chapitre conceptuel pour introduire une perspective chronologique avec l'évocation de l'émergence de la problématique transnationaliste et d'autre part, en inscrivant sa réflexion dans une problématique urbaine, il réinvestit la question de l'appartenance - ouverte au cours du second chapitre - en développant une approche spatialisée des objets de recherche que sont les minorités et l'ethnicité.

L'économie générale de l'ouvrage permet au public non familier du thème de prendre connaissance d'une notion qui par bien des aspects reste avant tout descriptive. Comme le souligne l'auteur après avoir pris le temps d'un travail de définition approfondi, le phénomène diasporique s'offre à la recherche comme une "notion encore incertaine ». Néanmoins, en prenant appui sur l'histoire ancienne du mot par sa connaissance des mondes juif et grec, Michel Bruneau dresse le parcours du mot diaspora et surtout de son sens. De cette notion judé- centrée, il montre avec efficacité à la fois le glissement sémantique qu'elle connaît au cours de son inscription dans le matériau théorique des sciences sociales mais aussi la difficulté progressive que revêt son utilisation avec le « succès » qu'elle rencontre à la fin des années quatre-vingt. Tout en ne manquant pas de signifier ce problème de perte de sens du fait d'une sur-utilisation du terme comme synonyme de dispersion, d'émigration massive, l'auteur souligne avec justesse l'enrichissement du débat autour des diasporas avec le tournant post-moderne. Prenant appui sur les travaux de Christine Chivallon sur la diaspora noire, il souligne le rôle des Cultural studies dans la plus-value théorique que connaît le débat scientifique resté jusqu'alors dans une enceinte historiciste et descriptive - par l'introduction d'une lecture fluide des identités éloignant ainsi le débat d'une tendance à l'essentialisation des groupes dits diasporiques. Couplée à l'exposé des éléments de problématique que suggère la question des diasporas lorsqu'elle est pensée comme miroir des changements que connaissent les Etats-Nations dans le contexte de globalisation, l'analyse de Michel Bruneau permet au lecteur de mieux saisir la diversité des lieux d'inscription de la notion de diaspora dans les problématiques structurant les disciplines comme la sociologie, les sciences politiques et bien sûr en géographie. Par cette notion, l'auteur parcourt l'ensemble des questionnements qui font sens aujourd'hui dans la recherche sur le couple migration-identité.

5 L'apport scientifique majeur de cet ouvrage réside dans sa volonté affirmée de démêler le nœud conceptuel des diasporas en déjouant le "tout diaspora», promu par les médias et les diasporas elles-mêmes, par le recours systématique à la diversité de la production scientifique et à la mise en écho des argumentations qui y sont développées. Ainsi les exemples arménien, chinois, indien, antillais, libanais, palestinien mais aussi tsigane, replacés dans différents contextes géographiques et politiques, permettent à l'auteur d'édifier un chemin de contention resserrant l'étau conceptuel sur les diasporas. Michel Bruneau parvient donc au fil des multiples appuis trouvés dans la pluralité des groupes étudiés à esquisser les contours de la notion de diasporas mais comme nous tous - l'auteur peine à saisir le concept même si l'argument d'une spécificité diasporique qui résiderait dans la capacité des groupes à se penser dans un territoire imaginé, fantasmé, retient nécessairement l'attention.

En termes conceptuels, l'apport de cet ouvrage à portée réflexive est à double détente : primo, grâce à la richesse des regards portés sur l'histoire des civilisations, il nous convainc de la surenchère théorique des travaux sur le transnationalisme; secundo, il soulève la question du biais heuristique des travaux récents sur le thème. Les analyses des phénomènes de dispersion, des rapports au territoire et des relations sociales en 
dispersion ou en exil ont-elles des finalités théoriques si différentes de celles suggérées par Thucydide ou Gerasimos Vlakhos cités en introduction. Sans vouloir caricaturer le propos, à la lecture de l'ouvrage, la question de l'apport théorique des recherches sur la notion de diaspora reste posée. Comme le soulignait Alessandro Monsutti dans un récent ouvrage à propos de la recherche sur le thème en anthropologie, il convient pour poursuivre la réflexion théorique engagée par de nombreux auteurs, comme ici Michel Bruneau, de savoir si nous parlons de «l'objet d'étude ou du regard».

\section{NOTES}

1. Stéphane Dufoix, 2003, Les Diasporas, Paris, PUF (Que sais-je ?).

\section{AUTEURS}

\section{WILLIAM BERTHOMIÈRE}

Migrinter, CNRS /Université de Poitiers 